\title{
RECURRENCE RELATIONS FOR SOBOLEV ORTHOGONAL POLYNOMIALS
}

\begin{abstract}
We consider recurrence relations for the polynomials orthonormal with respect to the Sobolev-type inner product and generated by classical orthogonal polynomials, namely: Jacobi polynomials, Legendre polynomials, Chebyshev polynomials of the first and the second kind, Gegenbauer (ultraspherical) polynomials, Hermite polynomials.
\end{abstract}

Key words: Sobolev orthogonal polynomials; recurrence relations; Jacobi polynomials; Legendre polynomials; Chebyshev polynomials; ultraspherical polynomials; Gegenbauer polynomials; Hermite polynomials; Laguerre polynomials; special polynomials

2010 Mathematical Subject Classification: 12E10, 26C05, $65 Q 30$

1. Introduction. Sobolev orthogonal polynomials were first considered in the early 1960's in relation with problems of approximation theory (e.g., [3]). In the last three decades, this new theory has been intensively developing (e.g., [1]-[22]). For more details on this topic, see the survey [14] and works cited there.

As the name implies, these polynomials are orthogonal with respect to the so-called Sobolev-type inner products. There are plenty of inner products of this kind, with various degrees of generalization. However, we only consider Sobolev-type inner products that can be represented as

$$
\langle f, g\rangle=\langle f, g\rangle_{W_{L_{\omega}^{2}}^{r}}=\sum_{\nu=0}^{r-1} f^{(\nu)}(a) g^{(\nu)}(a)+\int_{a}^{b} f^{(r)}(t) g^{(r)}(t) \omega(t) d t .
$$

For this case, the theory of Sobolev orthogonal polynomials has recently been significantly developed and has found important applications (see [22] and works cited there).

(C) Petrozavodsk State University, 2020 
A distinctive property of inner products of this kind is the existence of special points, such that the behavior of Sobolev orthogonal functions can be "controlled" in their neighborhood. Due to this property, it is possible to construct the Fourier series with respect to the Sobolev orthogonal polynomials, so that partial sums coincide with the approximated function at the ends of the orthogonality segment. Such series proved to be a convenient tool for different applied tasks, such as representing solutions of the Cauchy problem for differential equations.

Following the established notation, we denote by $L_{\omega}^{p}(a, b)$ the space of functions $f(x)$, measurable on $(a, b)$, for which $\int_{a}^{b}|f(x)|^{p} \omega(x) d x<\infty$, where $\omega=\omega(x)$ is a weight function.

Let $\left\{\varphi_{n}\right\}_{n=0}^{\infty}$ be a system of polynomials, orthonormal in $L_{\omega}^{2}(a, b)$. In other words,

$$
\left\langle\varphi_{n}, \varphi_{m}\right\rangle_{L_{\omega}^{2}}=\int_{a}^{b} \varphi_{n}(t) \varphi_{m}(t) \omega(t) d t=\delta_{n, m}
$$

where $\delta_{n, m}$ is the Kronecker symbol.

By $W_{L_{\omega}^{2}(a, b)}^{r}$ we denote the Sobolev space, which consists of functions $f=f(x)$ that are continuously differentiable $(r-1)$-times on $[a, b]$, while $f^{(r-1)}(x)$ is absolutely continuous on $[a, b]$ and $f^{(r)} \in L_{\omega}^{2}(a, b)$. The inner product in $W_{L_{\omega}^{2}(a, b)}^{r}$ is defined by the equality (1).

Sharapudinov I. I. proposed a new method for construction of polynomials orthogonal with respect to the inner product (1). For any given orthogonal system $\left\{\varphi_{n}(x)\right\}$, we can generate Sobolev orthogonal system using the following equations:

$$
\begin{gathered}
\varphi_{r, n}(x)=\frac{(x-a)^{n}}{n !}, \quad n=0,1, \ldots, r-1, \\
\varphi_{r, r+n}(x)=\frac{1}{(r-1) !} \int_{a}^{x}(x-t)^{r-1} \varphi_{n}(t) d t, \quad n=0,1, \ldots,
\end{gathered}
$$

hereinafter we will consider $\varphi_{0, n}(x)=\varphi_{n}(x)$. More precisely, the following statement has been proven in [18]. 
Theorem A Suppose that the functions $\varphi_{n}(x)(n=0,1, \ldots)$ form a complete orthonormal system in $L_{\omega}^{2}(a, b)$. Then the system $\left\{\varphi_{r, n}(x)\right\}$, generated by the system $\left\{\varphi_{n}(x)\right\}$ by means of the equalities (2) and (3), is complete in $W_{L_{\omega}^{2}(a, b)}^{r}$ and orthonormal with respect to the inner product (1).

Remark. Note that Theorem $A$ is valid not only for the case when $\varphi_{n}(x)$ are polynomials, but for more general case of orthogonal functions.

One of the key properties of orthogonal polynomials is the three-term recurrence relation, which establishes the relation between $n$-th polynomial and two previous $(n-1)$-th and $(n-2)$-th (for example, see $\S 3.2$, Theorem 3.2.1 in [24]):

$$
\varphi_{n}(x)=\left(A_{n} x+B_{n}\right) \varphi_{n-1}(x)+C_{n} \varphi_{n-2}(x), \quad n=2,3, \ldots .
$$

This formula is not only used for calculation of polynomial value in any given point $x$ for any degree $n$, but also for investigation of further properties of polynomial system.

As it was mentioned in [14], one of the main difficulties in the development of the Sobolev orthogonal polynomials theory is absence of three-terms recurrence relation for these polynomials in the general case. However, we managed to establish recurrence relations for the case of Sobolev-type inner products, which can be represented as (1).

In this article, we also consider specific Sobolev orthonormal polynomials generated by the classic orthonormal polynomials and establish recurrence relations for these polynomials (namely, for polynomials generated by: Jacobi polynomials, Legendre polynomials, Chebyshev polynomials of the first and second kind, Gegenbauer (ultraspherical) polynomials, Hermite polynomials). Recurrence relations for the Sobolev-Laguerre polynomials were established in [6], we only give them in the last section to cover this topic in more details.

2. Recurrence relations for Sobolev orthogonal polynomials. In the current section, we establish recurrence relations for Sobolev orthonormal polynomials $\left\{\varphi_{r, n}\right\}$ in the general case.

First, for the case when $n<r$ it is obvious that

$$
\varphi_{r, 0}(x)=1, \varphi_{r, n}(x)=\frac{(x-a)^{n}}{n !}=\frac{x-a}{n} \varphi_{r, n-1}(x), n=1, \ldots, r-1 .
$$

Next, from (3) with $n=0$, we get 


$$
\begin{aligned}
\varphi_{r, r}(x)=\frac{1}{(r-1) !} \int_{a}^{x}(x-t)^{r-1} \varphi_{0} d t=-\left.\frac{\varphi_{0}}{r !}(x-t)^{r}\right|_{a} ^{x} d t= \\
=\varphi_{0} \frac{(x-a)^{r}}{r !}=\frac{x-a}{r} \varphi_{r-1, r-1}(x), \quad r=1,2, \ldots,
\end{aligned}
$$

while $\varphi_{0,0}(x)=\varphi_{0}$.

Finally, for the case of $\varphi_{r, r+n}(x)(n \geq 1)$, from (4) and again from (3) we have

$$
\begin{aligned}
& \varphi_{r, r+n}(x)=\frac{1}{(r-1) !} \int_{a}^{x}(x-t)^{r-1} \varphi_{n}(t) d t= \\
& =\frac{1}{(r-1) !} \int_{a}^{x}(x-t)^{r-1}\left(\left(A_{n} t+B_{n}\right) \varphi_{n-1}(t)+C_{n} \varphi_{n-2}(t)\right) d t= \\
& =B_{n} \varphi_{r, r+n-1}(x)+C_{n} \varphi_{r, r+n-2}(x)+\frac{A_{n}}{(r-1) !} \int_{a}^{x}(x-t)^{r-1} t \varphi_{n-1}(t) d t .
\end{aligned}
$$

Let us consider the last term separately:

$$
\begin{gathered}
\frac{A_{n}}{(r-1) !} \int_{a}^{x}(x-t)^{r-1} t \varphi_{n-1}(t) d t=\frac{A_{n}}{(r-1) !} \int_{a}^{x}(x-t)^{r-1}(t-x+x) \varphi_{n-1}(t) d t= \\
=\frac{A_{n} x}{(r-1) !} \int_{a}^{x}(x-t)^{r-1} \varphi_{n-1}(t) d t-\frac{A_{n} r}{r !} \int_{a}^{x}(x-t)^{r} \varphi_{n-1}(t) d t= \\
=A_{n} x \varphi_{r, r+n-1}(x)-A_{n} r \varphi_{r+1, r+n}(x) .
\end{gathered}
$$

Collecting together equations (5) - (8) we get

Theorem 1. For the Sobolev orthogonal system $\left\{\varphi_{r, n}\right\}(r \geq 1)$, generated by the orthonormal system $\left\{\varphi_{n}\right\}$, the following recurrence relations hold:

$$
\begin{gathered}
\varphi_{r, 0}(x)=1, \quad \varphi_{r, n}(x)=\frac{x-a}{n} \varphi_{r, n-1}(x), \quad 1 \leq n \leq r-1 \\
\varphi_{0,0}(x)=\varphi_{0}, \quad \varphi_{r, r}(x)=\frac{x-a}{r} \varphi_{r-1, r-1}(x),
\end{gathered}
$$




$$
\begin{aligned}
& A_{n} r \varphi_{r+1, r+n}(x)=\left(A_{n} x+B_{n}\right) \varphi_{r, r+n-1}(x)+ \\
& +C_{n} \varphi_{r, r+n-2}(x)-\varphi_{r, r+n}(x), \quad n \geq 2,
\end{aligned}
$$

where $A_{n}, B_{n}$, and $C_{n}$ are the coefficients from the three-term recurrent relation (4) for the original system $\left\{\varphi_{n}\right\}$.

Remark. Theorem 1 does not cover the case of $\varphi_{1, n+1}(x)$, i. e.,

$$
\varphi_{1, n+1}(x)=\int_{a}^{x} \varphi_{n}(t) d t, \quad n=1,2, \ldots
$$

It should be considered separately, using special properties of the original orthogonal system $\left\{\varphi_{n}\right\}$ (such as integral and differential properties).

3. Some information about the Jacobi polynomials. For arbitrary real $\alpha$ and $\beta$, the Jacobi polynomials $P_{n}^{\alpha, \beta}(x)$ can be determined using the Rodrigues formula:

$$
P_{n}^{\alpha, \beta}(x)=\frac{(-1)^{n}}{2^{n} n !} \frac{1}{\rho(x)} \frac{d^{n}}{d x^{n}}\left\{\rho(x) \sigma^{n}(x)\right\},
$$

where $\rho(x)=\rho(x ; \alpha, \beta)=(1-x)^{\alpha}(1+x)^{\beta}, \sigma(x)=1-x^{2}$.

We will need the following properties of Jacobi polynomials [24], [23]:

$$
\begin{gathered}
P_{n}^{\alpha, \beta}(-x)=(-1)^{n} P_{n}^{\beta, \alpha}(x), \\
P_{n}^{\alpha, \beta}(-1)=(-1)^{n}\left(\begin{array}{c}
n+\beta \\
n
\end{array}\right), \quad P_{n}^{\alpha, \beta}(1)=\left(\begin{array}{c}
n+\alpha \\
n
\end{array}\right), \\
\frac{d}{d x} P_{n}^{\alpha, \beta}(x)=\frac{1}{2}(n+\alpha+\beta+1) P_{n-1}^{\alpha+1, \beta+1}(x), \\
\left(\begin{array}{c}
n \\
l
\end{array}\right) P_{n}^{\alpha,-l}(x)=\left(\begin{array}{c}
n+\alpha \\
l
\end{array}\right)\left(\frac{x+1}{2}\right)^{l} P_{n-l}^{\alpha, l}(x), \quad 1 \leq l \leq n, \\
(1-x) P_{n}^{\alpha+1, \beta}(x)+(1+x) P_{n}^{\alpha, \beta+1}(x)=2 P_{n}^{\alpha, \beta}(x), \\
P_{n}^{\alpha, \beta-1}(x)-P_{n}^{\alpha-1, \beta}(x)=P_{n-1}^{\alpha, \beta}(x), \\
(2 n+\alpha+\beta) P_{n}^{\alpha-1, \beta}(x)=(n+\alpha+\beta) P_{n}^{\alpha, \beta}(x)-(n+\beta) P_{n-1}^{\alpha, \beta}(x), \\
(2 n+\alpha+\beta) P_{n}^{\alpha, \beta-1}(x)=(n+\alpha+\beta) P_{n}^{\alpha, \beta}(x)+(n+\alpha) P_{n-1}^{\alpha, \beta}(x) .
\end{gathered}
$$


From (13) we also derive

$$
P_{n+1}^{-1,-1}(x)=\frac{x^{2}-1}{4} P_{n-1}^{1,1}(x) .
$$

Lemma 1. The following equalities for the Jacobi polynomials hold:

$$
\begin{gathered}
P_{n}^{\alpha-1, \beta-1}(x)=\frac{n+\lambda}{2 n+\lambda} P_{n}^{\alpha, \beta}(x)-\left(x-\frac{\alpha-\beta}{2 n+\lambda}\right) \frac{1}{2} P_{n-1}^{\alpha, \beta}(x)= \\
=\left(x+\frac{\alpha-\beta}{2 n+\lambda-2}\right) \frac{n+\lambda-1}{2 n} P_{n-1}^{\alpha, \beta}(x)-\frac{(n+\alpha-1)(n+\beta-1)}{n(2 n+\lambda-2)} P_{n-2}^{\alpha, \beta}(x),
\end{gathered}
$$

where $\lambda=\alpha+\beta$.

Proof. Using (14), (15) and (17) we get

$$
\begin{aligned}
& 2 P_{n}^{\alpha-1, \beta-1}(x)=(1-x) P_{n}^{\alpha, \beta-1}(x)+(1+x) P_{n}^{\alpha-1, \beta}(x)= \\
&=2 P_{n}^{\alpha, \beta-1}(x)-\left[P_{n}^{\alpha, \beta-1}(x)-P_{n}^{\alpha-1, \beta}(x)\right]-x\left[P_{n}^{\alpha, \beta-1}(x)-P_{n}^{\alpha-1, \beta}(x)\right]= \\
&=2\left[\frac{n+\alpha+\beta}{2 n+\alpha+\beta} P_{n}^{\alpha, \beta}(x)+\frac{1}{2}\left(\frac{\alpha-\beta}{2 n+\alpha+\beta}-x\right) P_{n-1}^{\alpha, \beta}(x)\right] .
\end{aligned}
$$

From the other hand, from (16) and (17) we deduce

$$
\begin{gathered}
P_{n}^{\alpha-1, \beta-1}(x)=\frac{n+\alpha+\beta-1}{2 n+\alpha+\beta-1} P_{n}^{\alpha, \beta-1}(x)-\frac{n+\beta-1}{2 n+\alpha+\beta-1} P_{n-1}^{\alpha, \beta-1}(x)= \\
=\frac{n+\alpha+\beta-1}{2 n+\alpha+\beta-1}\left[\frac{n+\alpha+\beta}{2 n+\alpha+\beta} P_{n}^{\alpha, \beta}(x)+\frac{n+\alpha}{2 n+\alpha+\beta} P_{n-1}^{\alpha, \beta}(x)\right]- \\
-\frac{n+\beta-1}{2 n+\alpha+\beta-1}\left[\frac{n+\alpha+\beta-1}{2 n+\alpha+\beta-2} P_{n-1}^{\alpha, \beta}(x)+\frac{n+\alpha-1}{2 n+\alpha+\beta-2} P_{n-2}^{\alpha, \beta}(x)\right]= \\
=\frac{n+\alpha+\beta-1}{2 n+\alpha+\beta}\left[\frac{n+\alpha+\beta}{2 n+\alpha+\beta-1} P_{n}^{\alpha, \beta}(x)+\frac{\alpha-\beta}{2 n+\alpha+\beta-2} P_{n-1}^{\alpha, \beta}(x)\right]- \\
-\frac{(n+\alpha-1)(n+\beta-1)}{(2 n+\alpha+\beta-2)(2 n+\alpha+\beta-1)} P_{n-2}^{\alpha, \beta}(x) .
\end{gathered}
$$

Consider the recurrence relation for Jacobi polynomials:

$$
\begin{aligned}
& 2(n+1)(n+\alpha+\beta+1)(2 n+\alpha+\beta) P_{n+1}^{\alpha, \beta}(x)= \\
= & {\left[(2 n+\alpha+\beta)(2 n+\alpha+\beta+2) x+\alpha^{2}-\beta^{2}\right](2 n+\alpha+\beta+1) P_{n}^{\alpha, \beta}(x)-}
\end{aligned}
$$




$$
-2(n+\alpha)(n+\beta)(2 n+\alpha+\beta+2) P_{n-1}^{\alpha, \beta}(x),
$$

and rewrite it in the following form:

$$
\begin{aligned}
\frac{n+\alpha+\beta}{2 n+\alpha+\beta-1} P_{n}^{\alpha, \beta}(x)= & {\left[\frac{2 n+\alpha+\beta}{2 n} x+\frac{\alpha^{2}-\beta^{2}}{2 n(2 n+\alpha+\beta-2)}\right] P_{n-1}^{\alpha, \beta}(x)-} \\
& -\frac{(n+\alpha-1)(n+\beta-1)(2 n+\alpha+\beta)}{n(2 n+\alpha+\beta-1)(2 n+\alpha+\beta-2)} P_{n-2}^{\alpha, \beta}(x) .
\end{aligned}
$$

Then, returning to the previous equality, we get

$$
\begin{aligned}
& P_{n}^{\alpha-1, \beta-1}(x)=\frac{n+\alpha+\beta-1}{2 n+\alpha+\beta}\left[\frac{n+\alpha+\beta}{2 n+\alpha+\beta-1} P_{n}^{\alpha, \beta}(x)+\right. \\
&+\left.\frac{\alpha-\beta}{2 n+\alpha+\beta-2} P_{n-1}^{\alpha, \beta}(x)\right]-\frac{(n+\alpha-1)(n+\beta-1)}{(2 n+\alpha+\beta-2)(2 n+\alpha+\beta-1)} P_{n-2}^{\alpha, \beta}(x)= \\
&= \frac{n+\alpha+\beta-1}{2 n+\alpha+\beta}\left[\frac{(2 n+\alpha+\beta)}{2 n} x+\frac{\alpha^{2}-\beta^{2}}{2 n(2 n+\alpha+\beta-2)}+\frac{\alpha-\beta}{2 n+\alpha+\beta-2}\right] \times \\
& \times P_{n-1}^{\alpha, \beta}(x)-\frac{(n+\alpha-1)(n+\beta-1)}{(2 n+\alpha+\beta-2)(2 n+\alpha+\beta-1)}\left[\frac{n+\alpha+\beta-1}{n}+1\right] P_{n-2}^{\alpha, \beta}(x)= \\
&= \frac{n+\alpha+\beta-1}{2 n}\left[x+\frac{\alpha-\beta}{2 n+\alpha+\beta-2}\right] P_{n-1}^{\alpha, \beta}(x)- \\
&-\frac{(n+\alpha-1)(n+\beta-1)}{n(2 n+\alpha+\beta-2)} P_{n-2}^{\alpha, \beta}(x) .
\end{aligned}
$$

The proof is complete.

If $\alpha, \beta>-1$, then the Jacobi polynomials form a complete orthogonal system in $L_{\rho}^{2}(-1,1)$, i. e.,

$$
\int_{-1}^{1} P_{n}^{\alpha, \beta}(t) P_{m}^{\alpha, \beta}(t) \rho(t) d t=h_{n}^{\alpha, \beta} \delta_{n m},
$$

where

$$
h_{n}^{\alpha, \beta}=\frac{\Gamma(n+\alpha+1) \Gamma(n+\beta+1) 2^{\alpha+\beta+1}}{n ! \Gamma(n+\alpha+\beta+1)(2 n+\alpha+\beta+1)} .
$$

Let $p_{n}^{\alpha, \beta}(x)=\left[h_{n}^{\alpha, \beta}\right]^{-1 / 2} P_{n}^{\alpha, \beta}(x)$ be the orthonormal Jacobi polynomials.

The following recurrence relation holds: 


$$
\begin{gathered}
\sqrt{\frac{4(n+1)(n+\alpha+1)(n+\beta+1)(n+\alpha+\beta+1)}{(2 n+\alpha+\beta+1)(2 n+\alpha+\beta+2)^{2}(2 n+\alpha+\beta+3)}} p_{n+1}^{\alpha, \beta}(x)= \\
=\left[x+\frac{\alpha^{2}-\beta^{2}}{(2 n+\alpha+\beta+2)(2 n+\alpha+\beta)}\right] p_{n}^{\alpha, \beta}(x)- \\
-\sqrt{\frac{4 n(n+\alpha)(n+\beta)(n+\alpha+\beta)}{(2 n+\alpha+\beta)^{2}(2 n+\alpha+\beta-1)(2 n+\alpha+\beta+1)}} p_{n-1}^{\alpha, \beta}(x), n \geq 1 .
\end{gathered}
$$

4. The Sobolev-Jacobi polynomials. Consider the polynomials $p_{r, n}^{\alpha, \beta}(x)(r=1,2, \ldots ; n=0,1,2, \ldots)$ defined on $[-1,1]$ by the equalities

$$
\begin{gathered}
p_{r, n}^{\alpha, \beta}(x)=\frac{(x+1)^{n}}{n !}, \quad n=0,1, \ldots, r-1, \\
p_{r, r+n}^{\alpha, \beta}(x)=\frac{1}{(r-1) !} \int_{-1}^{x}(x-t)^{r-1} p_{n}^{\alpha, \beta}(t) d t, \quad n=0,1, \ldots
\end{gathered}
$$

We will restrict the parameters $\alpha, \beta$ to satisfy the inequality $\alpha, \beta>-1$ since this is the only case when the Jacobi polynomials are orthogonal and, hence, the three-term recurrence relation still holds.

For brevity, denote $\lambda=\alpha+\beta$.

1. In the case $r=1$, we have

$$
p_{1, n+1}^{\alpha, \beta}(x)=\int_{-1}^{x} p_{n}^{\alpha, \beta}(t) d t=\frac{1}{\sqrt{h_{n}^{\alpha, \beta}}} \int_{-1}^{x} P_{n}^{\alpha, \beta}(t) d t, \quad n=1,2, \ldots
$$

From (12), we get

$$
\frac{2}{n+\lambda} \frac{d}{d x} P_{n+1}^{\alpha-1, \beta-1}(x)=P_{n}^{\alpha, \beta}(x)
$$

then

$$
\begin{aligned}
p_{1, n+1}^{\alpha, \beta}(x)= & \frac{2\left(h_{n}^{\alpha, \beta}\right)^{-\frac{1}{2}}}{n+\lambda} \int_{-1}^{x} \frac{d}{d t} P_{n+1}^{\alpha-1, \beta-1}(t) d t= \\
& =\frac{2\left(h_{n}^{\alpha, \beta}\right)^{-\frac{1}{2}}}{n+\lambda}\left[P_{n+1}^{\alpha-1, \beta-1}(x)-P_{n+1}^{\alpha-1, \beta-1}(-1)\right]=
\end{aligned}
$$




$$
=\frac{2\left(h_{n}^{\alpha, \beta}\right)^{-\frac{1}{2}}}{n+\lambda}\left[P_{n+1}^{\alpha-1, \beta-1}(x)+(-1)^{n}\left(\begin{array}{c}
n+\beta \\
n+1
\end{array}\right)\right], \quad n=1,2, \ldots
$$

Note, that we need the parameters $\alpha, \beta$ to be non-negative to use (26) as a recurrence relation; otherwise, the polynomial $P_{n+1}^{\alpha-1, \beta-1}(x)$ would not be orthogonal, and, hence, the recurrence relation for it would not hold. For the case $-1 \leq \alpha, \beta \leq 0$, we can use (19) or (20) from Lemma 1. We get

$$
\begin{aligned}
& p_{1, n+1}^{\alpha, \beta}(x)=\frac{\left(h_{n}^{\alpha, \beta}\right)^{-\frac{1}{2}}}{n+\lambda}\left[\frac{2(n+\lambda+1)}{2 n+\lambda+2} P_{n+1}^{\alpha, \beta}(x)-\right. \\
& \left.-\left(x-\frac{\alpha-\beta}{2 n+\lambda+2}\right) P_{n}^{\alpha, \beta}(x)+(-1)^{n} 2\left(\begin{array}{c}
n+\beta \\
n+1
\end{array}\right)\right]= \\
& =\frac{\left(h_{n}^{\alpha, \beta}\right)^{-\frac{1}{2}}}{n+\lambda} \frac{2(n+\lambda+1)}{2 n+\lambda+2}\left[\begin{array}{c}
P_{n+1}^{\alpha, \beta}(x)-\left(x-\frac{\lambda x+\alpha-\beta}{2(n+\lambda+1)}\right) P_{n}^{\alpha, \beta}(x)- \\
\left.\quad-P_{n+1}^{\alpha, \beta}(-1)-\frac{n+\alpha+1}{n+\lambda+1} P_{n}^{\alpha, \beta}(-1)\right] .
\end{array}\right.
\end{aligned}
$$

Using the fact that

$$
h_{n}^{\alpha, \beta}=\frac{4(n+1)}{n+\lambda} h_{n+1}^{\alpha-1, \beta-1},
$$

we can also rewrite (26) in the following form:

$$
p_{1, n+1}^{\alpha, \beta}(x)=\tilde{a}_{n}^{\alpha, \beta} p_{n+1}^{\alpha-1, \beta-1}(x)+\tilde{b}_{n}^{\alpha, \beta},
$$

where

$$
\tilde{a}_{n}^{\alpha, \beta}=\frac{1}{\sqrt{(n+1)(n+\lambda)}}, \quad \tilde{b}_{n}^{\alpha, \beta}=(-1)^{n} \frac{2\left(h_{n}^{\alpha, \beta}\right)^{-\frac{1}{2}}}{n+\lambda}\left(\begin{array}{c}
n+\beta \\
n+1
\end{array}\right) .
$$

2. Next, we consider the case $r \geq 2$. First, let us rewrite the recurrence relation (23) in the following form:

$$
\begin{gathered}
p_{n}^{\alpha, \beta}(x)=\left(a_{n}^{\alpha, \beta} x+b_{n}^{\alpha, \beta}\right) p_{n-1}^{\alpha, \beta}(x)+c_{n}^{\alpha, \beta} p_{n-2}^{\alpha, \beta}(x), \quad n=2,3, \ldots, \\
a_{n}^{\alpha, \beta}=\frac{1}{2} \sqrt{\frac{(2 n+\lambda-1)(2 n+\lambda)^{2}(2 n+\lambda+1)}{n(n+\alpha)(n+\beta)(n+\lambda)}},
\end{gathered}
$$




$$
\begin{gathered}
b_{n}^{\alpha, \beta}=\frac{1}{2} \frac{\alpha^{2}-\beta^{2}}{(2 n+\lambda-2)} \sqrt{\frac{(2 n+\lambda-1)(2 n+\lambda+1)}{n(n+\alpha)(n+\beta)(n+\lambda)}}, \\
c_{n}^{\alpha, \beta}=\frac{-(2 n+\lambda)}{2 n+\lambda-2} \sqrt{\frac{(n-1)(n+\alpha-1)(n+\beta-1)(n+\lambda-1)(2 n+\lambda+1)}{n(n+\alpha)(n+\beta)(n+\lambda)(2 n+\lambda-3)}} .
\end{gathered}
$$

From Theorem 1, we have

$$
\begin{aligned}
& a_{n}^{\alpha, \beta} r p_{r+1, r+n}^{\alpha, \beta}(x)=\left(a_{n}^{\alpha, \beta} x+b_{n}^{\alpha, \beta}\right) p_{r, r+n-1}^{\alpha, \beta}(x)+ \\
& \quad+c_{n}^{\alpha, \beta} p_{r, r+n-2}^{\alpha, \beta}(x)-p_{r, r+n}^{\alpha, \beta}(x), \quad r \geq 1, n \geq 2 .
\end{aligned}
$$

Dividing both parts of the equation by $a_{n}^{\alpha, \beta}$ and performing simple transformations, we get

$$
\begin{aligned}
r p_{r+1, r+n}^{\alpha, \beta}(x)= & \left(x+A_{n}^{\alpha, \beta}\right) p_{r, r+n-1}^{\alpha, \beta}(x)- \\
& -B_{n-1}^{\alpha, \beta} p_{r, r+n-2}^{\alpha, \beta}(x)-B_{n}^{\alpha, \beta} p_{r, r+n}^{\alpha, \beta}(x), \quad r \geq 1, n \geq 2,
\end{aligned}
$$

where

$$
\begin{gathered}
A_{n}^{\alpha, \beta}=\frac{b_{n}^{\alpha, \beta}}{a_{n}^{\alpha, \beta}}=\frac{\alpha^{2}-\beta^{2}}{(2 n+\lambda-2)(2 n+\lambda)}, \\
B_{n}^{\alpha, \beta}=\frac{1}{a_{n}^{\alpha, \beta}}=\frac{c_{n+1}^{\alpha, \beta}}{a_{n+1}^{\alpha, \beta}}=\frac{2}{2 n+\lambda} \sqrt{\frac{n(n+\alpha)(n+\beta)(n+\lambda)}{(2 n+\lambda-1)(2 n+\lambda+1)}} .
\end{gathered}
$$

Using (26), (27), and (29) from Theorem 1, we derive the following result. Theorem 2. For the Sobolev-Jacobi polynomials $\left\{p_{r, n}^{\alpha, \beta}\right\}(r \geq 1)$, when $\alpha, \beta>-1$, the following recurrence relations hold:

$$
\begin{gathered}
p_{r, 0}^{\alpha, \beta}(x)=1, \quad p_{r, n}^{\alpha, \beta}(x)=\frac{x+1}{n} p_{r, n-1}^{\alpha, \beta}(x), \quad 1 \leq n \leq r-1 ; \\
p_{0,0}^{\alpha, \beta}(x)=\frac{1}{\sqrt{h_{0}^{\alpha, \beta}}}, \quad p_{r, r}^{\alpha, \beta}(x)=\frac{x+1}{r} p_{r-1, r-1}^{\alpha, \beta}(x) ; \\
p_{1, n+1}^{\alpha, \beta}(x)=\frac{2\left(h_{n}^{\alpha, \beta}\right)^{-\frac{1}{2}}}{n+\lambda}\left[P_{n+1}^{\alpha-1, \beta-1}(x)-P_{n+1}^{\alpha-1, \beta-1}(-1)\right]= \\
=\frac{\left(h_{n}^{\alpha, \beta}\right)^{-\frac{1}{2}}}{n+\lambda} \frac{2(n+\lambda+1)}{2 n+\lambda+2}\left[P_{n+1}^{\alpha, \beta}(x)-\left(x-\frac{\lambda x+\alpha-\beta}{2(n+\lambda+1)}\right) P_{n}^{\alpha, \beta}(x)-\right.
\end{gathered}
$$




$$
\begin{gathered}
\left.-P_{n+1}^{\alpha, \beta}(-1)-\frac{n+\alpha+1}{n+\lambda+1} P_{n}^{\alpha, \beta}(-1)\right] ; \\
r p_{r+1, r+n}^{\alpha, \beta}(x)=\left(x+A_{n}^{\alpha, \beta}\right) p_{r, r+n-1}^{\alpha, \beta}(x)- \\
\quad-B_{n-1}^{\alpha, \beta} p_{r, r+n-2}^{\alpha, \beta}(x)-B_{n}^{\alpha, \beta} p_{r, r+n}^{\alpha, \beta}(x), \quad n \geq 2,
\end{gathered}
$$

where $\lambda=\alpha+\beta$,

$$
\begin{gathered}
A_{n}^{\alpha, \beta}=\frac{\alpha^{2}-\beta^{2}}{(2 n+\lambda-2)(2 n+\lambda)}, \\
B_{n}^{\alpha, \beta}=\frac{2}{2 n+\lambda} \sqrt{\frac{n(n+\alpha)(n+\beta)(n+\lambda)}{(2 n+\lambda-1)(2 n+\lambda+1)}} .
\end{gathered}
$$

5. The Sobolev-Gegenbauer polynomials. If parameters $\alpha$ and $\beta$ of the Jacobi polynomial are equal, then this polynomial is called ultrasphrerical polynomial. The special case of ultrasphrerical polynomials when $\alpha=\beta=\gamma-\frac{1}{2}$ are Gegenbauer polynomials. The relation between Gegenbauer and standartized Jacobi polynomials is established by the following equality:

$$
C_{n}^{\gamma}(x)=\frac{\Gamma\left(\gamma+\frac{1}{2}\right)}{\Gamma(2 \gamma)} \frac{\Gamma(n+2 \gamma)}{\Gamma\left(n+\gamma+\frac{1}{2}\right)} P_{n}^{\gamma-\frac{1}{2}, \gamma-\frac{1}{2}}(x) .
$$

Consider orthonormal Gegenbauer polynomials

$$
\hat{C}_{n}^{\gamma}(x)=p_{n}^{\gamma-\frac{1}{2}, \gamma-\frac{1}{2}}(x), \quad \gamma>-\frac{1}{2}, \quad n=0,1,2, \ldots
$$

We can generate the new Sobolev orthogonal system from $\hat{C}_{n}^{\gamma}(x)$ :

$$
C_{r, n}^{\gamma}(x)=p_{r, n}^{\gamma-\frac{1}{2}, \gamma-\frac{1}{2}}(x)
$$

with the help of equalities (24) and (25).

Using (26) and (30), we can write the following for these polynomials:

$$
C_{1, n+1}^{\gamma}(x)=p_{1, n+1}^{\gamma-\frac{1}{2}, \gamma-\frac{1}{2}}(x)=\frac{2\left(h_{n}^{\gamma-\frac{1}{2}, \gamma-\frac{1}{2}}\right)^{-\frac{1}{2}}}{n+2 \gamma-1}\left[P_{n+1}^{\gamma-\frac{3}{2}, \gamma-\frac{3}{2}}(x)-\right.
$$




$$
\left.-P_{n+1}^{\gamma-\frac{3}{2}, \gamma-\frac{3}{2}}(-1)\right]=\frac{\Gamma(\gamma-1)}{2^{1-\gamma}} \sqrt{\frac{(n+\gamma) n !}{2 \pi \Gamma(n+2 \gamma)}}\left[C_{n+1}^{\gamma-1}(x)-C_{n+1}^{\gamma-1}(-1)\right] .
$$

This formula is suitable for the case when $\gamma>\frac{1}{2}$. On the other hand, from (27) and, once again, (30) we get

$$
\begin{aligned}
& C_{1, n+1}^{\gamma}(x)=\frac{\left(h_{n}^{\gamma-\frac{1}{2}, \gamma-\frac{1}{2}}\right)^{-\frac{1}{2}}}{n+2 \gamma-1} \frac{2(n+2 \gamma)}{2 n+2 \gamma+1}\left[P_{n+1}^{\gamma-\frac{1}{2}, \gamma-\frac{1}{2}}(x)-\right. \\
& \left.-P_{n+1}^{\gamma-\frac{1}{2}, \gamma-\frac{1}{2}}(-1)-\frac{2 n+2 \gamma+1}{2(n+2 \gamma)}\left(x P_{n}^{\gamma-\frac{1}{2}, \gamma-\frac{1}{2}}(x)+P_{n}^{\gamma-\frac{1}{2}, \gamma-\frac{1}{2}}(-1)\right)\right]= \\
& =\frac{2^{\gamma} \Gamma(\gamma) \sqrt{(n+\gamma) n !}}{(n+2 \gamma-1) \sqrt{2 \pi \Gamma(n+2 \gamma)}}\left[C_{n+1}^{\gamma}(x)-x C_{n}^{\gamma}(x)-C_{n+1}^{\gamma}(-1)-C_{n}^{\gamma}(-1)\right]
\end{aligned}
$$

From these two equalities and Theorem 2, we conclude

Corollary 1. For the Sobolev-Gegenbauer polynomials $\left\{C_{r, n}^{\gamma}\right\}(r \geq 1)$, when $\gamma>-\frac{1}{2}$, the following recurrence relations hold:

$$
\begin{gathered}
C_{r, 0}^{\gamma}(x)=1, \quad C_{r, n}^{\gamma}(x)=\frac{x+1}{n} C_{r, n-1}^{\gamma}(x), \quad 1 \leq n \leq r-1 ; \\
C_{0,0}^{\gamma}(x)=\frac{1}{\sqrt{h_{0}^{\gamma-\frac{1}{2}, \gamma-\frac{1}{2}}}}=\sqrt{\frac{\Gamma(\gamma+1)}{\sqrt{\pi} \Gamma\left(\gamma+\frac{1}{2}\right)}}, \quad C_{r, r}^{\gamma}(x)=\frac{x+1}{r} C_{r-1, r-1}^{\gamma}(x) ; \\
C_{1, n+1}^{\gamma}(x)=2^{\gamma-1} \Gamma(\gamma-1) \sqrt{\frac{(n+\gamma) n !}{2 \pi \Gamma(n+2 \gamma)}}\left[C_{n+1}^{\gamma-1}(x)-C_{n+1}^{\gamma-1}(-1)\right]= \\
=\frac{2^{\gamma} \Gamma(\gamma) \sqrt{(n+\gamma) n !}}{(n+2 \gamma-1) \sqrt{2 \pi \Gamma(n+2 \gamma)}}\left[C_{n+1}^{\gamma}(x)-x C_{n}^{\gamma}(x)-\right. \\
\left.-C_{n+1}^{\gamma}(-1)-C_{n}^{\gamma}(-1)\right], \quad n=1,2, \ldots ; \\
r C_{r+1, r+n}^{\gamma}(x)=x C_{r, r+n-1}^{\gamma}(x)-B_{n-1}^{\gamma} C_{r, r+n-2}^{\gamma}(x)-B_{n}^{\gamma} C_{r, r+n}^{\gamma}(x), \quad n \geq 2, \\
\text { where } \\
B_{n}^{\gamma}=\sqrt{\frac{n(n+2 \gamma-1)}{4(n+\gamma-1)(n+\gamma)}} .
\end{gathered}
$$


6. The Sobolev-Legendre polynomials. In case when parameters $\alpha=\beta=0$, the Jacobi polynomials become the well-known Legendre polynomials $P_{n}(x)$. Using the orthonormal Legendre polynomials

$$
\hat{P}_{n}(x)=p_{n}^{0,0}(x), \quad n=0,1,2, \ldots,
$$

we generate the Sobolev-Legendre polynomials

$$
p_{r, n}(x)=p_{r, n}^{0,0}(x) .
$$

From (28) for $r=1$, we have

$$
p_{1, n+1}(x)=\frac{p_{n+1}^{-1,-1}(x)}{\sqrt{n(n+1)}}=\frac{\sqrt{2(2 n+1)}}{n} P_{n+1}^{-1,-1}(x), \quad n=1,2, \ldots
$$

Using (18), we rewrite

$$
p_{1, n+1}(x)=\frac{\sqrt{2 n+1}}{2 n \sqrt{2}}\left(x^{2}-1\right) P_{n-1}^{1,1}(x), \quad n=1,2, \ldots .
$$

Then, from this equality and from Theorem 2, we get

Corollary 2. For the Sobolev-Legendre polynomials $p_{r, n}(x)(r \geq 1)$, the following recurrence relations hold:

$$
\begin{gathered}
p_{r, 0}(x)=1, \quad p_{r, n}(x)=\frac{x+1}{n} p_{r, n-1}(x), \quad 1 \leq n \leq r-1 ; \\
p_{r, r}(x)=\frac{1}{\sqrt{h_{0}^{0,0}}}=\frac{1}{\sqrt{2}}, \quad p_{r, r}(x)=\frac{x+1}{r} p_{r-1, r-1}(x) ; \\
p_{1, n+1}(x)=\frac{\sqrt{2 n+1}}{2 n \sqrt{2}}\left(x^{2}-1\right) P_{n-1}^{1,1}(x), \quad n=1,2, \ldots ; \\
r p_{r+1, r+n}(x)=x p_{r, r+n-1}(x)-B_{n-1}^{\frac{1}{2}} p_{r, r+n-2}(x)-B_{n}^{\frac{1}{2}} p_{r, r+n}(x), \quad n \geq 2,
\end{gathered}
$$

where

$$
B_{n}^{\frac{1}{2}}=\frac{n}{\sqrt{4 n^{2}-1}}
$$

7. The Sobolev-Chebyshev polynomials. The next two important special cases of the Jacobi polynomials are the Chebyshev polynomials of the first and the second kind. 
1. Orthonormal Chebyshev polynomials of the first kind can be written in trigonometric form as follows:

$$
\hat{T}_{0}=\frac{1}{\sqrt{\pi}}, \quad \hat{T}_{n}(x)=\sqrt{\frac{2}{\pi}} \cos n \arccos (x), \quad n=1,2, \ldots
$$

These polynomials are orthonormal on $[-1,1]$ with the weight function $\frac{1}{\sqrt{1-x^{2}}}$, so

$$
\hat{T}_{n}(x)=p_{n}^{-\frac{1}{2},-\frac{1}{2}}(x) .
$$

Then Sobolev-Chebyshev polynomials of the first kind will be

$$
\begin{gathered}
\hat{T}_{r, n}(x)=\frac{(x+1)^{n}}{n !}, \quad n=0,1, \ldots, r-1 ; \quad \hat{T}_{r, r}(x)=\frac{(x+1)^{r}}{r ! \sqrt{\pi}} \\
\hat{T}_{r, r+n}(x)=\frac{1}{(r-1) !} \int_{-1}^{x}(x-t)^{r-1} \hat{T}_{n}(t) d t, \quad n=1,2, \ldots
\end{gathered}
$$

In the work [18] by Sharapudinov I. I., the asymptotic formula and some other properties of the modified Sobolev-Chebyshev polynomials of the first kind were considered (orthonormal with the weight function $\frac{2}{\pi \sqrt{1-x^{2}}}$ ).

In particular, the recurrence relations for the case $r=1$ were obtained. We will use them here, transforming for our case:

$$
\begin{aligned}
& \hat{T}_{1, n+1}(x)=\frac{\hat{T}_{n+1}(x)}{2(n+1)}-\frac{\hat{T}_{n-1}(x)}{2(n-1)}-\frac{(-1)^{n}}{n^{2}-1}, \quad n \geq 2 ; \\
& \hat{T}_{1,0}(x)=1, \quad \hat{T}_{1,1}(x)=\frac{x+1}{\sqrt{\pi}}, \quad \hat{T}_{1,2}(x)=\frac{x^{2}-1}{\sqrt{2 \pi}} .
\end{aligned}
$$

Then, from Theorem 2 we get

Corollary 3. For the Sobolev-Chebyshev polynomials of the first kind $\hat{T}_{r, n}(x)(r \geq 1)$ the following recurrence relations hold:

$$
\begin{gathered}
\hat{T}_{r, 0}(x)=1, \quad \hat{T}_{r, n}(x)=\frac{x+1}{n} \hat{T}_{r, n-1}(x), \quad 1 \leq n \leq r-1 ; \\
\hat{T}_{0,0}(x)=\hat{T}_{0}=\frac{1}{\sqrt{\pi}}, \quad \hat{T}_{r, r}(x)=\frac{(x+1)}{r} \hat{T}_{r-1, r-1}(x) ; \\
\hat{T}_{1,0}(x)=1, \quad \hat{T}_{1,1}(x)=\frac{x+1}{\sqrt{\pi}}, \quad \hat{T}_{1,2}(x)=\frac{x^{2}-1}{\sqrt{2 \pi}},
\end{gathered}
$$




$$
\begin{gathered}
\hat{T}_{1, n+1}(x)=\frac{\hat{T}_{n+1}(x)}{2(n+1)}-\frac{\hat{T}_{n-1}(x)}{2(n-1)}-\frac{(-1)^{n}}{n^{2}-1}, \quad n \geq 2 ; \\
\hat{T}_{r+1, r+n}(x)=\frac{x}{r} \hat{T}_{r, r+n-1}(x)-\frac{1}{2 r}\left[\hat{T}_{r, r+n-2}(x)+\hat{T}_{r, r+n}(x)\right], \quad n \geq 2 .
\end{gathered}
$$

2. Classical Chebyshev polynomials of the second kind can be written in trigonometric form as follows:

$$
U_{n}(x)=\frac{\sin (n+1) \arccos x}{\sin \arccos x}, \quad n=0,1,2, \ldots
$$

It is easy to show that

$$
\int_{-1}^{1} U_{n}(x) U_{m}(x) \sqrt{1-x^{2}} d x=\frac{\pi}{2} \delta_{n m} .
$$

Hence, orthonormal Chebyshev polynomials of the second kind are

$$
\widehat{U}_{n}(x)=\sqrt{\frac{2}{\pi}} U_{n}(x)=p_{n}^{\frac{1}{2}, \frac{1}{2}}(x) .
$$

Sobolev - Chebyshev polynomials of the second kind are of the following form:

$$
\begin{gathered}
U_{r, n}(x)=\frac{(x+1)^{n}}{n !}, \quad n=0,1, \ldots, r-1, \\
U_{r, r+n}(x)=\frac{\sqrt{2 / \pi}}{(r-1) !} \int_{-1}^{x}(x-t)^{r-1} U_{n}(t) d t, \quad n=0,1, \ldots
\end{gathered}
$$

By its definition, we have $U_{1,0}(x)=1$; and from (31) we get

$$
U_{1,1}(x)=\sqrt{\frac{2}{\pi}}(x+1), \quad U_{1,2}(x)=\sqrt{\frac{2}{\pi}}\left(x^{2}-1\right) .
$$

Using the following well-known properties of Chebyshev polynomials:

$$
\int U_{n}(t) d t=\frac{T_{n+1}(t)}{n+1}, \quad T_{n}(-1)=(-1)^{n},
$$

from (31) we also deduce

$$
U_{1, n}(x)=\frac{1}{n} \sqrt{\frac{2}{\pi}}\left[T_{n}(x)-(-1)^{n}\right] .
$$


With the help of these equalities, we derive the next statement from Theorem 2:

Corollary 4. For the Sobolev-Chebyshev polynomials of the second kind $U_{r, n}(x)(r \geq 1)$ the following recurrence relations hold:

$$
\begin{gathered}
U_{r, 0}(x)=1, \quad U_{r, n}(x)=\frac{x+1}{n} U_{r, n-1}(x), \quad 1 \leq n \leq r-1 ; \\
U_{0,0}(x)=\hat{U}_{0}=\sqrt{\frac{2}{\pi}}, \quad U_{r, r}(x)=\frac{x+1}{r} U_{r-1, r-1}(x) ; \\
U_{1,0}(x)=1, \quad U_{1,1}(x)=\sqrt{\frac{2}{\pi}}(x+1), \quad U_{1,2}(x)=\sqrt{\frac{2}{\pi}}\left(x^{2}-1\right), \\
U_{1, n}(x)=\frac{1}{n} \sqrt{\frac{2}{\pi}}\left[T_{n}(x)-(-1)^{n}\right], \quad n \geq 3 ; \\
U_{r+1, r+n}(x)=\frac{x}{r} U_{r, r+n-1}(x)-\frac{1}{2 r}\left[U_{r, r+n}(x)+U_{r, r+n-2}(x)\right], \quad n \geq 2 .
\end{gathered}
$$

8. Some information about the Hermite polynomials. The standardized Hermite (or Chebyshev-Hermite) polynomials are determined using the following Rodrigues formula (see [23], [24]):

$$
H_{n}(x)=(-1)^{n} e^{x^{2}} \frac{d^{n}}{d x^{n}}\left\{e^{-x^{2}}\right\}, \quad n=0,1,2, \ldots
$$

These polynomials are orthogonal with the even weight function $h(t)=e^{-t^{2}}$, defined on the whole real axis; namely:

$$
\int_{-\infty}^{\infty} H_{n}(t) H_{m}(t) h(t) d t=\delta_{n m} 2^{n} n ! \sqrt{\pi} .
$$

Therefore,

$$
\widehat{H}_{n}(t)=\frac{H_{n}(t)}{\sqrt{2^{n} n ! \sqrt{\pi}}}, \quad n=0,1,2, \ldots,
$$

are orthonormal Hermite polynomials.

The three-term recurrence relation for Hermite polynomials has the following form:

$$
\begin{gathered}
H_{0}(x)=1, \quad H_{1}(x)=2 x \\
H_{n}(x)=2 x H_{n-1}(x)-2(n-1) H_{n-2}(x), \quad n \geq 2 .
\end{gathered}
$$


Hence for orthonormal polynomials we have

$$
\begin{gathered}
\widehat{H}_{0}(x)=\pi^{-1 / 4}, \quad \widehat{H}_{1}(x)=x \sqrt{2} \pi^{-1 / 4}, \\
\widehat{H}_{n}(x)=x \widehat{H}_{n-1}(x) \sqrt{\frac{2}{n}}-\widehat{H}_{n-2}(x) \sqrt{\frac{n-1}{n}}, \quad n \geq 2 .
\end{gathered}
$$

We will also need the following properties of the Hermite polynomials (see [23]):

$$
\begin{gathered}
H_{n}^{\prime}(x)=2 n H_{n-1}(x) ; \\
H_{2 n}(0)=(-1)^{n} \frac{(2 n) !}{n !}, \quad H_{2 n+1}(0)=0 .
\end{gathered}
$$

9. The Sobolev-Hermite polynomials. The following SobolevHermite polynomials were considered in the work [19]:

$$
\begin{gathered}
h_{r, n}(x)=\frac{x^{n}}{n !}, \quad n=0,1, \ldots, r-1, \\
h_{r, r+n}(x)=\frac{1}{(r-1) !} \int_{0}^{x}(x-t)^{r-1} \hat{H}_{n}(t) d t, \quad n=0,1, \ldots
\end{gathered}
$$

It has been shown, that these polynomials form the complete system in the Sobolev space $W_{L_{h}^{2}(\mathbb{R})}^{r}$ and are orthonormal with respect to the following inner product:

$$
\langle f, g\rangle=\sum_{\nu=0}^{r-1} f^{(\nu)}(0) g^{(\nu)}(0)+\int_{\mathbb{R}} f^{(r)}(t) g^{(r)}(t) h(t) d t .
$$

Let us consider recurrence relations for Sobolev-Hermite polynomials.

1. Using (34), we have, for the case $r=1$ :

$$
\begin{aligned}
h_{1, n+1}(x)=\int_{0}^{x} \hat{H}_{n}(t) d t=\frac{1}{\sqrt{2^{n} n ! \sqrt{\pi}}} \int_{0}^{x} \frac{1}{2(n+1)} H_{n+1}^{\prime}(t) d t= \\
=\frac{H_{n+1}(x)-H_{n+1}(0)}{\sqrt{2^{n+2}(n+1) ! \sqrt{\pi}(n+1)}} .
\end{aligned}
$$

If $n$ is even $(n=2 m)$, this equation is simplified, with the help of (35), to

$$
h_{1,2 m+1}(x)=\frac{H_{2 m+1}(x)}{2^{m+1}(2 m+1) \sqrt{(2 m) ! \sqrt{\pi}}}, \quad m=0,1, \ldots,
$$


while for odd $n=2 m+1$ we get

$$
h_{1,2 m+2}(x)=\frac{H_{2 m+2}(x)+(-1)^{m} \frac{(2 m+2) !}{(m+1) !}}{2^{m+2}(m+1) \sqrt{2(2 m+1) ! \sqrt{\pi}}}, \quad m=0,1, \ldots
$$

2. For the case $r \geq 2$, we use Theorem 1 and deduce:

$$
\begin{aligned}
\tilde{A}_{n} r h_{r+1, r+n}(x)= & \left(\tilde{A}_{n} x+\tilde{B}_{n}\right) h_{r, r+n-1}(x)+ \\
& +\tilde{C}_{n} h_{r, r+n-2}(x)-h_{r, r+n}(x), \quad r \geq 1, n \geq 2,
\end{aligned}
$$

where $\tilde{A}_{n}, \tilde{B}_{n}$ and $\tilde{C}_{n}$ are given by recurrence relation (33); hence

$$
\begin{aligned}
& r h_{r+1, r+n}(x)=x h_{r, r+n-1}(x)- \\
&-\sqrt{\frac{n-1}{2}} h_{r, r+n-2}(x)-\sqrt{\frac{n}{2}} h_{r, r+n}(x), \quad r \geq 1, n \geq 2 .
\end{aligned}
$$

Collecting all the formulas together, we get the following from Theorem 1:

Theorem 3. For the Sobolev-Hermite polynomials $h_{r, n}(x)(r \geq 1)$ the following recurrence relations hold:

$$
\begin{gathered}
h_{r, 0}(x)=1, \quad h_{r, n}(x)=\frac{x}{n} h_{r, n-1}(x), \quad 1 \leq n \leq r-1 ; \\
h_{0,0}(x)=\hat{H}_{0}=\pi^{-1 / 4}, \quad h_{r, r}(x)=\frac{x}{r} h_{r-1, r-1}(x) ; \\
h_{1,2 m}(x)=\frac{1}{2^{m+1} \sqrt{m(2 m) ! \sqrt{\pi}}}\left[H_{2 m}(x)-(-1)^{m} \frac{(2 m) !}{m !}\right], \quad m=1,2, \ldots, \\
h_{1,2 m+1}(x)=\frac{H_{2 m+1}(x)}{2^{m+1}(2 m+1) \sqrt{(2 m) ! \sqrt{\pi}}}, \quad m=1,2, \ldots ; \\
h_{r+1, r+n}(x)=\frac{x}{r} h_{r, r+n-1}(x)-\sqrt{\frac{n-1}{2 r}} h_{r, r+n-2}(x)-\sqrt{\frac{n}{2 r}} h_{r, r+n}(x), n \geq 2 .
\end{gathered}
$$

10. The Sobolev-Laguerre polynomials. The Laguerre polynomials can be defined using the Rodrigues formula (see [23], [24]):

$$
L_{n}^{\alpha}(x)=\frac{1}{n !} x^{-\alpha} e^{x} \frac{d^{n}}{d x^{n}}\left\{x^{n+\alpha} e^{-x}\right\}
$$


When $\alpha>-1$, these polynomials are orthogonal with respect to the following inner product:

$$
\int_{0}^{\infty} L_{n}^{\alpha}(t) L_{m}^{\alpha}(t) t^{\alpha} e^{-t} d t=\delta_{n m}\left(\begin{array}{c}
n+\alpha \\
n
\end{array}\right) \Gamma(\alpha+1) .
$$

We denote by $l_{n}^{\alpha}(x)$ the orthonormal version of these polynomials.

Now, we can generate (see [19]) the new system

$$
\begin{gathered}
l_{r, n}^{\alpha}(x)=\frac{x^{n}}{n !}, \quad n=0,1, \ldots, r-1, \\
l_{r, r+n}^{\alpha}(x)=\frac{1}{(r-1) !} \int_{0}^{x}(x-t)^{r-1} l_{n}^{\alpha}(t) d t, \quad n=0,1, \ldots,
\end{gathered}
$$

which is complete in the Sobolev space $W_{L_{\rho}^{2}(0, \infty)}^{r}$ and orthonormal with respect the Sobolev-type inner product

$$
\langle f, g\rangle=\sum_{\nu=0}^{r-1} f^{(\nu)}(0) g^{(\nu)}(0)+\int_{0}^{\infty} f^{(r)}(t) g^{(r)}(t) \rho(t) d t,
$$

where $\rho=\rho(t)=t^{\alpha} e^{-t}$.

The following recurrence relations were established in [6]:

Theorem B For the Sobolev-Laguerre polynomials $l_{r, n}^{\alpha}(x)(r \geq 1)$, when $\alpha>-1$, the following recurrence relations hold:

$$
\begin{gathered}
l_{r, 0}^{\alpha}(x)=1, \quad l_{r, n}^{\alpha}(x)=\frac{x}{n} l_{r, n-1}^{\alpha}(x), \quad 1 \leq n \leq r-1 ; \\
l_{0,0}^{\alpha}(x)=l_{0}^{\alpha}=\frac{1}{\sqrt{\Gamma(\alpha+1)}}, \quad l_{r, r}^{\alpha}(x)=\frac{x}{r} l_{r-1, r-1}^{\alpha}(x) ; \\
-\sqrt{\frac{n+\alpha+1}{n+1}} l_{n+1}^{\alpha}(x)+l_{n}^{\alpha}(x)+\sqrt{\frac{n+\alpha+1}{n+1}} l_{n+1}^{\alpha}(x)=l_{n}^{\alpha}(0), \quad n \geq 1 ; \\
b_{n}^{\alpha} r l_{r+1, r+n}^{\alpha}(x)= \\
l_{r, r+n}^{\alpha}(x)+\left[b_{n}^{\alpha} x-a_{n}^{\alpha}\right] l_{r, r+n-1}^{\alpha}(x)+c_{n}^{\alpha} l_{r, r+n-2}^{\alpha}(x), \quad n \geq 2,
\end{gathered}
$$

where

$$
a_{n}^{\alpha}=\frac{2 n+\alpha-1}{\sqrt{n(n+\alpha)}}, \quad b_{n}^{\alpha}=\frac{1}{\sqrt{n(n+\alpha)}}, \quad c_{n}^{\alpha}=\sqrt{\frac{(n-1)(n+\alpha-1)}{n(n+\alpha)}} .
$$


Remark. Equations (37) and (38) can also be represented as follows:

$$
l_{1, n+1}^{\alpha}(x)=l_{n}^{\alpha}(x)-\sqrt{\frac{n+\alpha+1}{n+1}} l_{n+1}^{\alpha}(x)+\left(\begin{array}{c}
n+\alpha \\
n
\end{array}\right)\left[\left(\frac{n+\alpha+1}{n+1}\right)^{\frac{3}{2}}-1\right]
$$

for $n \geq 1$;

$$
r l_{r+1, r+n}^{\alpha}(x)=\tilde{a}_{n}^{\alpha} l_{r, r+n}^{\alpha}(x)+\left[x-\tilde{b}_{n}^{\alpha}\right] l_{r, r+n-1}^{\alpha}(x)+\tilde{a}_{n-1}^{\alpha} l_{r, r+n-2}^{\alpha}(x)
$$

for $n \geq 2$, where

$$
\tilde{a}_{n}^{\alpha}=\sqrt{n(n+\alpha)}, \quad \tilde{b}_{n}^{\alpha}=2 n+\alpha-1 .
$$

\section{References}

[1] Aktas R., Xu Y. Sobolev orthogonal polynomials on a simplex. Int. Math. Res. Notice, 2013, no. 13, pp. 3087-3131.

DOI: https://doi.org/10.1093/imrn/rns141

[2] Alfaro M., Moreno-Balcázar J. J., Peña A., Rezola M. L. A new approach to the asymptotics of Sobolev type orthogonal polynomials. J. Approx. Theory, 2011, vol. 163, pp. 460-480.

DOI:https://doi.org/10.1016/j.jat.2010.11.005

[3] Althammer P. Eine Erweiterung des Orthogonalitatsbegriffes bei Polynomen und deren Anwendung auf die beste Approximation. J. Reine Angew. Math., 1962, vol. 211, pp. 192-204.

DOI: https://doi.org/10.1515/crll.1962.211.192

[4] Berti A. C., Bracciali C. F., Sri Ranga A. Orthogonal polynomials associated with related measures and Sobolev orthogonal polynomials. Numer. Algorithms, 2003, vol. 34, pp. 203-216.

DOI: https://doi.org/10.1023/B:NUMA.0000005363.32764.d3

[5] Díaz Mendoza C., Orive R., Pijeira Cabrera H. Zeros and logarithmic asymptotics of Sobolev orthogonal polynomials for exponential weights. J. Comput. Appl. Math., 2009, vol. 233, pp. 691-698.

DOI: https://doi.org/10.1016/j.cam.2009.02.037

[6] Gadzhimirzaev R. M. Recurrence relations for polynomials orthonormal on Sobolev, generated by Laguerre polynomials. Izv. Saratov Univ. (N.S.), Ser. Math. Mech. Inform., 2018, vol. 18:1, pp. 17-24.

DOI: https://doi .org/10.18500/1816-9791-2018-18-1-17-24 
[7] Geronimo J. S., Lubinsky D. S., Marcellán F. Asymptiotic for Sobolev orthogonal polynomials for exponential weights. Constr. Approx., 2005, vol. 22 , pp. 309-346.

DOI: https://doi.org/10.1007/s00365-004-0578-1

[8] Iserles A., Koch P. E., Norsett S. P. and Sanz-Serna J. M. On polynomials orthogonal with respect to certain Sobolev inner products. J. Approx. Theory, 1991, vol. 65 , pp. 151-175.

DOI: https://doi.org/10.1016/0021-9045(91)90100-0

[9] Kwon K. H., Littlejohn L. L. Sobolev orthogonal polynomials and secondorder differential equations. Rocky Mountain J. Math., 1998, vol. 28, pp. 547-594.

DOI: https://doi.org/10.1216/rmjm/1181071786

[10] Lee J. K. and Littlejohn L. L. Sobolev orthogonal polynomials in two variables and second order partial differential equations. J. Math. Anal. Appl., 2006, vol. 322, pp. 1001-1017.

DOI: https://doi.org/10.1016/j.jmaa.2005.09.062

[11] López G., Marcellán F., Van Assche W. Relative Asymptotics for Polynomials Orthogonal with Respect to a Discrete Sobolev Inner-Product. Constr. Approx., 1995, vol. 11:1, pp. 107-137.

DOI: https://doi.org/10.1007/BF01294341

[12] Marcellán F., Alfaro M. and Rezola M. L. Orthogonal polynomials on Sobolev spaces: old and new directions. Journal of Computational and Applied Mathematics, 1993, vol. 48, pp. 113-131.

DOI: https://doi .org/10.1016/0377-0427 (93)90318-6

[13] Marcellán F., Zejnullahu R. Xh., Fejzullahu B. Xh., Huertas E. On orthogonal polynomials with respect to certain discrete Sobolev inner product. Pacific J. of Math., 2012, vol. 257, pp. 167-188.

DOI: https://doi.org/10.2140/pjm.2012.257.167

[14] Marcellán F., Xu Y. On Sobolev orthogonal polynomials. Expositiones Mathematicae, 2015, vol. 33:3, pp. 308-352.

DOI: https://doi.org/10.1016/j. exmath.2014.10.002

[15] Meijer H. G. Laguerre polynimials generalized to a certain discrete Sobolev inner product space. J. Approx. Theory, 1993, vol. 73, pp. 1-16.

DOI: https://doi.org/10.1006/jath.1993.1029

[16] Pérez T. E., Piñar M. A. and Xu Y. Weighted Sobolev orthogonal polynomials on the unit ball. Int. Math. Res. Notice, 2013, no. 13, pp. 3087-3131. J. Approx. Theory, 2013, vol. 171, pp. 84-104.

DOI: https://doi.org/10.1016/j.jat.2013.03.004 
[17] Ruiz H. D., Marcellán F., Molano A. Asymptotics of Sobolev orthogonal polynomials for Hermite $(1,1)$-coherent pairs. J. of Mathematical Analysis and Applications, 2018, vol. 467, iss. 1, pp. 601-621.

DOI: https://doi.org/10.1016/j.jmaa.2018.06.075

[18] Sharapudinov I. I. Asymptotic properties of polynomials, orthogonal in Sobolev sense and associated with the Jacobi polynomials. Daghestan Electronic Mathematical Reports, 2016, vol. 6, pp. 1-24.

DOI: https://doi.org/10.31029/demr.6.1

[19] Sharapudinov I. I., Gadzhieva Z. D., Gadzhimirzaev R. M. Systems of functions orthogonal with respect to scalar products of Sobolev type with discrete masses generated by classical orthogonal systems. Daghestan Electronic Mathematical Reports, 2016, vol. 6, pp. 31-60.

DOI: https://doi.org/10.31029/demr.6.3

[20] Sharapudinov I. I. Special series in Laguerre polynomials and their approximation properties. Sib. Math. J., 2017, vol 58, pp. 338-362.

DOI: https://doi.org/10.1134/S0037446617020173

[21] Sharapudinov I. I. Sobolev-orthogonal systems of functions associated with an orthogonal system. Izvestiya: Mathematics, 2018, vol. 82:1, pp. 212-244. DOI: http://dx.doi.org/10.1070/IM8536

[22] Sharapudinov I. I. Sobolev-orthogonal systems of functions and some of their applications. Russian Math. Surveys, 2019, vol. 74:4, pp. 659-733. DOI: https://doi.org/10.4213/rm9846

[23] Suetin P.K. Classic orthogonal polynomials. M.: Fizmatlit, 2005, 480 p.

[24] Szego G. Orthogonal Polynomials. AMS Colloq. Publ, 1939, vol. 23.

DOI: https://doi.org/10.1090/coll/023

Received November 26, 2019.

In revised form, April 19, 2020.

Accepted May 02, 2020.

Published online May 20, 2020.

Dagestan Federal Research Center of RAS

45, M.Gadzhieva st., Makhachkala, 367000, Russia

E-mail: Sultanakhmedov@gmail.com 